\title{
Rebecca Horn, corps sous tension
}

Rebecca Horn, Körper unter Spannung

Rebecca Horn, Turning the Body On

\section{Léa Barbisan}

\section{CpenEdition}

\section{Journals}

Édition électronique

URL : https://journals.openedition.org/ceg/10144

DOI : $10.4000 /$ ceg. 10144

ISSN : 2605-8359

\section{Éditeur}

Presses Universitaires de Provence

Édition imprimée

Date de publication : 20 janvier 2020

Pagination : 201-212

ISBN : 979-10-320-0253-7

ISSN : 0751-4239

\section{Référence électronique}

Léa Barbisan, «Rebecca Horn, corps sous tension », Cahiers d'Études Germaniques [En ligne], 78 | 2020, mis en ligne le 21 octobre 2021, consulté le 01 février 2022. URL : http://journals.openedition.org/ceg/ 10144 ; DOI : https://doi.org/10.4000/ceg.10144 


\section{Rebecca Horn, corps sous tension}

Léa BARBISAN

Sorbonne-Université, SIRICE (UMR 8138), Paris

L'œuvre de Rebecca Horn ( $\left.{ }^{*} 1944\right)$ frappe d'abord par son caractère protéiforme. L'artiste allemande se fait connaître, dès la fin des années 1960, par ses créations de prothèses - des " extensions corporelles " qu'elle harnache aux corps d'ami.e.s artistes ou à son propre corps et qu'elle mobilise dans des performances d'abord intimistes, puis de plus en plus exposées. En 1972, Harald Szeemann l'invite à présenter l'une de ces performances à la Documenta 5. C'est là l'une des premières consécrations de son travail, qui se diversifie par la suite dans ses formes, ses matériaux et ses médiums : les performances font progressivement place à la réalisation de moyens et de longs métrages, aux installations in situ, à l'écriture de poèmes, à la réalisation de dessins et de sculptures cinétiques.

Des prothèses que l'artiste nomme " extensions corporelles " aux sculptures cinétiques, le chemin parcouru est long. Sur ce chemin, le corps humain semble s'être perdu : alors qu'il est partie prenante de l'œuvre de jeunesse, indispensable à la réalisation des performances, il disparaît dans les installations et les sculptures pour faire place à de minutieux mécanismes. Tout au plus subsiste-t-il sous forme de traces qui donnent à voir son absence : dans les installations qui forment le Kafkazyklus (Cycle Kafka, 1994), par exemple, de petites machines s'animent au-dessus de souliers, de parapluies et de jumelles abandonnés, soigneusement préservés dans des vitrines de verre. Dans ce monde où il ne reste de l'humanité que des vestiges à archiver, les machines semblent avoir capté toute l'énergie vitale : sur la scène que leur offre Horn, elles se meuvent, chuchotent à l'oreille des spectateurs, s'attirent et s'entre-tuent. Pourtant, cette disparition de la figure humaine n'indique pas de changement d'objet : Rebecca Horn ne se détourne pas du vivant au profit de l'inorganique. Elle poursuit bel et bien son travail sur le corps, mais sur un corps qu'elle ne conçoit d'emblée pas comme le support d'une identité stable, serait-elle celle de l'individu, du genre ou de l'espèce.

Chez elle, en effet, le corps est un espace en tension, une brèche par laquelle communiquent et s'hybrident des ordres que l'on a coutume de distinguer. Ces ordres, dont la philosophie moderne s'est attachée à penser la différence, sont ceux du sujet et de l'objet. Cette distinction fondamentale se décline en un ensemble de polarités : l'ego et le monde, le volontaire et l'involontaire, l'animé et l'inanimé. C'est précisément dans ces partitions - dans ces répartitions - que le corps vient jeter le trouble : car le corps n'est ni le " moi ", puisque c'est une entité matérielle qui prend sa place dans le monde empirique, ni un simple objet. 
La phénoménologie, depuis Husserl, explore la nature double du corps, cette " chose matérielle d'une certaine nature qui n'est pas simplement une chose matérielle, mais qui [...] constitue une pièce maîtresse pour la donnée de l'âme et de l'ego ${ }^{1} "$. On lui doit la conceptualisation de la distinction entre Körper, le corps-objet, la " chose matérielle ", et Leib, le corps-sujet, le corps propre, soit la " pièce maitresse pour la donnée de l'âme et de l'ego ". La phénoménologie husserlienne dédouble le corps pour lui donner sa place de chaque côté de la frontière entre le " moi " et l'autre. Rebecca Horn ne prend pas ce parti : elle choisit de montrer que le corps ne respecte pas la ligne de partage entre Körper et Leib, entre la chose et le corps vivant-vécu, entre le corps-objet et le corps reconnu comme " propre " par le sujet.

De ses premières performances à ses sculptures cinétiques récentes, l'artiste propose un travail cohérent où le corps se fait le lieu d'une transgression - d'un passage de frontière entre l'intime et l'étranger, l'humain et le non-humain, le vivant et l'artificiel -, qui s'effectue par la mise au jour des mouvements élémentaires, répétitifs et incoercibles, qui apparentent l'être humain à l'animal et à la machine. En mettant au cœur de son œuvre l'" impropre "de l'homme - cette dynamique animale, machinale qui anime les corps - Horn affirme la valeur esthétique de ces mouvements réflexes et pulsionnels longtemps jugés indignes d'accéder à la représentation artistique.

\section{Animal prothétique}

Les premiers opérateurs de cette transgression anthropologique et esthétique sont les " extensions corporelles " que Rebecca Horn crée dès la fin des années 1960. "Extensions corporelles " : le terme indique qu'il ne s'agit pas de sculptures, mais bien de prothèses qui modifient la perception des frontières du corps propre $^{2}$. L'une des plus connues fut construite pour la performance Einhorn (Licorne, 1970-72), dans laquelle une jeune femme nue harnachée et coiffée d'une longue corne blanche se frayait un chemin à l'aube dans les champs et les bois. Cette performance s'est révélée inaugurale à bien des égards : d'abord parce qu'elle se présente comme un jeu sur le nom de sa créatrice, qui fait de sa signature, légèrement altérée, l'idée et le titre de l'œuvre et revendique ainsi son statut d'artiste. Mais aussi parce que s'y manifeste la volonté de faire de l'" extension corporelle " l'agent d'une métamorphose : c'est là un enjeu

1. Edmund Husserl, Idées directrices pour une phénoménologie et une philosophie phénoménologique pures, Livre second. Recherches phénoménologiques pour la constitution, traduction d'Éliane Escoubas, Paris, PUF, 1996, p. 222.

2. À cet égard, le travail de Horn fait écho aux réflexions sur la prothèse menées par les théoriciens de la technique et des média - de Ernst Kapp, qui, dès la fin du XIX ${ }^{\mathrm{e}}$ siècle, fait des outils une " projection d'organes " (" Organprojektion ", dans Principes d'une philosophie de la technique, traduction de Grégoire Chamayou, Paris, Vrin, 2007, p. 199), à Marshall McLuhan, qui affirme, dans les années 1960, que " toutes les inventions ou technologies sont des prolongements ou autoamputations de nos corps " (Pour comprendre les médias. Les prolongements technologiques de l'homme, traduction de Jean Paré, Montréal, éditions HMH, 1968, p. 63). 
décisif des performances de Horn. La métamorphose est double : la créatrice et la performeuse, par l'adjonction d'un nouvel élément, deviennent licornes - Horn dans le titre de l'œuvre, la performeuse dans l'œuvre elle-même. L'objectif de l'" extension corporelle " est bien d'initier une métamorphose par laquelle le corps sublimé atteint au statut d'œuvre d'art. Cette sublimation, toutefois, est ambivalente, car si les " extensions corporelles " promettent un épanouissement du corps dans l'espace, elles induisent en même temps une réduction de la liberté de mouvement et un appauvrissement des sensations. Ainsi, dans Einhorn, la performeuse rappelle la licorne non tant par sa silhouette - la corne est trop massive, rien n'évoque la morphologie du cheval -, que par sa démarche bridée : le harnais qui enserre son torse, la corne en équilibre sur le sommet de sa tête la forcent à adopter un pas compassé, qui évoque la foulée du cheval domestiqué. La raideur du corps harnaché rappelle qu'un port altier est toujours l'effet de procédés de contrôle et de censure : la jeune femme - licorne errante, mais bel et bien captive - ne peut devenir animal fabuleux qu'au prix de sa liberté3.

Les " extensions corporelles " jouent toujours des effets troublants que génère la tension entre le déploiement et la privation. La prothèse "Handschuhfinger " (" doigts-gants "), constituée de cinq longs appendices que la performeuse - il s'agit ici de Horn - attache au bout de ses doigts dans la performance de 1974 Mit beiden Händen gleichzeitig die Wände berühren (Toucher les murs avec les deux mains à la fois), rend manifeste la duplicité de l'" extension corporelle " : si d'un côté elle allonge les doigts à l'extrême, leur donne une allure surréelle, arachnéenne, elle ne permet plus la saisie fine et supprime les sensations tactiles. Ironiquement, le gain se change en perte : enfiler les " doigts-gants ", c'est certes se donner les moyens de relever le défı de " toucher les murs avec les deux mains à la fois ", mais c'est aussi renoncer au tact et au contact. La performeuse fait d'inlassables allers et retours dans une pièce dans laquelle elle est seule à pouvoir prendre place : le spectateur n'assiste pas à un jeu, comme le titre semblerait le suggérer, mais se voit offrir l'image de la pénitence d'un Sisyphe roulant son rocher. Les " extensions corporelles " sont des armures derrière lesquelles le corps se barricade, ce sont aussi des harnais qui l'encombrent, des lanières qui l'enserrent - et l'envahissement de l'espace

3. Anette Kruszynski indique que le harnais de Einhorn est une référence à l'autoportrait de Frida Kahlo La colonne brisée (1944) : " Dieses Werk [...] zeigt die Künstlerin in einem orthopädischen Korsett, also eingeschnürt in ein unbewegliches Mieder, das beim Betrachter über Mitgefühl hinaus unwillkürlich ein Bedürfnis nach Befreiung auszulösen vermag " (Anette Kruszynski, " "Und verstehe die Freiheit, Aufzubrechen, wohin er will”. Autonomie und Befreiung im Schaffen von Rebecca Horn ", in Rebecca Horn. Bodylandscapes, Ostfildern, Hatje Cantz, 2004, p. 136-143, ici p. 136). Pour Alexandra Tacke, au contraire, la réinterprétation de ce motif proposée par Horn offre l'image d'une émancipation : " der hohe, selbstbewusste und feste Gang der Akteurin wird durch das lange, weiße Horn betont, macht aus ihr eine phallische Frau " (Alexandra Tacke, Rebecca Horn. Künstlerische Selbstpositionierungen im kulturellen Raum, Köln, Böhlau, 2011, p. 64). Si la corne massive de Einhorn évoque bien un symbole phallique, il nous semble que l'émancipation de la " licorne " de Horn n'est que partielle et, en ce sens, ambivalente, car l'imposant " phallus " harnaché sur tête de la performeuse la grandit autant qu'il l'encombre. 
va toujours de pair avec le cloisonnement du corps et l'appauvrissement des possibilités sensorielles et motrices. L'évolution des " extensions corporelles " et des performances qui s'y rattachent porte témoignage de cette ambivalence. Dans la performance Hahnenmaske (Masque de coq, 1973), l'artiste porte un masque vertical de plumes de coq noires qui lui barre le visage en deux par la hauteur. Ici aussi, l'" extension corporelle " fait obstacle au contact avec l'autre être humain, un homme au visage nu, dont la performeuse s'approche sans jamais s'autoriser à le toucher directement. Le masque maintient la distance entre les corps, force à choisir entre l'échange des regards et la caresse, et rend le baiser, la parole, la mimique impossibles.

C'est précisément la mise à distance, le maintien obstiné de la frontière entre un corps humain et l'autre qui permet, à un autre niveau, un passage de frontière transgressif. Horn, en s'armant de ses prothèses, se force à renoncer au " propre de l'homme ", à ce qui constitue son humanité : l'expressivité fine du visage, l'expression langagière, la précision du geste, la caresse et le baiser - pour retrouver des mouvements et des comportements primitifs, qui rapprochent l'être humain de l'animal. Dans Hahnenmaske, l'empêchement de la communication humaine permet de s'apparenter au coq. Horn décrit la performance en ces termes : " Je tourne lentement mon visage vers la personne en face de moi et je commence à caresser son visage avec les plumes douces du masque en profil. L'espace entre nos visages est entièrement rempli de plumes. Elles obstruent aussi ma vue. Je ne vois le visage face à moi que si je tourne ma tête d'un côté et le regarde d'un œil, comme un oiseau ${ }^{4}$. " Dans la performance Blinzeln (Cligner de l'œil, 1974), Horn pousse cette tentative de rapprochement à son comble : elle se filme en compagnie d'un cacatoès, dont elle imite le comportement. Le choix d'imiter l'oiseau, l'un des animaux les moins expressifs selon les critères humains, n'est pas anodin, il impose à la performeuse de ne recourir qu'à un répertoire minimal de mouvements - claquements répétitifs de la langue, clignements de l'œil. Ceux-ci nous mettent face à la réduction extrême des ressources expressives du corps humain, sur lesquelles reposent d'habitude les pratiques artistiques. Cette performance, qui ne procure aucune satisfaction esthétique, trouble la spectatrice partagée entre la fascination hypnotique et l'ennui. Alors que dans les performances précédentes, les " extensions corporelles " jouent de l'équilibre entre l'extravagance de la parure et la simplicité du mouvement, entre la surprise et la répétition, Blinzeln, l'une des rares performances dépourvues d'" extensions ", assume sa pauvreté. Cet " art pauvre " permet néanmoins à Horn de mettre en relief un petit ensemble de mouvements, d'ordinaire négligés parce qu'ils sont, justement, involontaires, immotivés et incoercibles - et ne s'inscrivent en ce sens dans aucun processus

4. "Slowly I turn my face to the person standing opposite me and begin to stroke them with my feathered profile. The feathers entirely fill the space between our faces and restrict my vision. I can only see the face opposite me when I turn my head to the side, and look with just one eye, like a bird ", Rebecca Horn (propos traduits), citée dans Carl Haenlein (dir.), Rebecca Horn. The Glance of Infinity, Zurich, Scalo, 1997, p. 65. 
créatif. Pourtant, ces mouvements réflexes sont des mouvements vitaux, que l'être humain a en partage avec les autres animaux, et qui l'inscrivent dans une communauté plus large que celle de l'espèce - communauté dont Rebecca Horn n'exclut pas non plus la machine. Plus encore qu'aux " extensions corporelles ", ce sont à ces mouvements primordiaux que s'attache Horn dans ses performances.

\section{Machinations de la pulsion}

Rebecca Horn place au cœur de son œuvre les réflexes, les automatismes somatiques qui portent notre vie, mais que nous ne reconnaissons pas comme nôtres, parce qu'ils n'expriment rien de nous, mais plutôt quelque chose de beaucoup plus primitif : une pulsation vitale. Freud parlait de " vexation du narcissisme humain ${ }^{5}$ " pour désigner les effets de chaque découverte scientifique qui forçait l'être humain à abandonner la position privilégiée de maîtrise qu'il s'était accordée : découverte de Copernic annonçant que la terre n'est pas au centre de l'univers; découverte de Darwin annonçant que l'être humain n'est pas une créature à part, mais un animal; découverte de Freud annonçant que le " moi " n'est pas maître chez lui, puisque l'essentiel des processus psychiques est inconscient. De la même manière, il y a un effet "blessant" dans le travail de Horn, qui contraint les performeurs à appauvrir leurs gestes jusqu'à renoncer à leur humanité, pour que se manifeste au sein de leur corps un mouvement animal, qui est, finalement, le mouvement machinal des organes - un mouvement premier, fondamental, jugé, d'ordinaire, indigne de toute mobilisation artistique, parce qu'il est exclusivement somatique. Mais, dès lors qu'il s'agit de mettre en lumière le pouvoir de fascination de l'automatisme corporel, le corps de la machine peut se substituer au corps humain pour exécuter ces performances élémentaires et captivantes. Les performances de Horn ne se contentent pas de renvoyer l'être humain à l'animal qui l'habite, elles le confrontent à la parenté entre le mouvement vital et le mouvement machinal. La performance Überströmer (Machine à couvrir de sang, 1970) met en scène la réversibilité des frontières entre le corps vivant et la machine : le corps nu du performeur est habillé d'un réseau de tuyaux où circule un liquide rouge. Horn écrit à propos de cette " extension corporelle " : " le sang est acheminé au moyen d'une pompe à travers les tubes en plastique qui passent par le contenant en verre; entourant son corps comme un habit de veines palpitantes, [Überströmer] force la personne immobile à devenir une extension du mécanisme lui-même ${ }^{6}$ ". Alors que le corps vivant

5. "Kränkung des menschlichen Narzissmus ", Sigmund Freud, Une difficulté de la psychanalyse, traduction de J. Altounian, A. Bourguignon, P. Cotet, A. Rauzy (1917), in CEuvres complètes, Paris, PUF, 1996, p. 47.

6. " Blood pumps, slowly, circulated through the glass container through the plastic tubes; enclosing his body like a pulsing garment of veins [it] forces the evolution of the motionless person into being an extension of the mechanism itself. " Rebecca Horn (propos traduits), http:// www.tate.org.uk/art/artworks/horn-overflowing-blood-machine-t07843 [dernière consultation le 14 juillet 2018]. 
s'immobilise, la machine prend en charge le mouvement vital : la prothèse n'est pas ici un accessoire qui vient suppléer à une défaillance corporelle ou accroître ses performances, c'est le corps qui devient une excroissance de la prothèse, un mécanisme auxiliaire qui soutient le fonctionnement de la machine. Cette performance annonce déjà la superfluité du corps humain.

Il n'est donc pas surprenant que le corps humain disparaisse progressivement des œuvres de Horn, pour être remplacé par des éléments naturels - plumes, ailes de papillon, minéraux - ou des objets articulés à des mécanismes qui les mettent en mouvement. Le mécanisme en effet, loin de se présenter comme l'opposé du mouvement organique, sert de révélateur à la pulsation vitale. Mais il ne donne pas à voir que les mouvements physiologiques les plus répétitifs : foulée de la marche, battement du cœur, clignement de l'œil, alternance de l'inspiration et de l'expiration. Rebecca Horn montre que ces mouvements essentiels de va-et-vient - qui sont à la base du fonctionnement de l'organisme comme de celui de la machine - ne sont pas simplement vitaux, mais qu'ils sont érotiques, parce qu'ils offrent aussi l'image du désir et de ses mécanismes, voire de ses machinations. Dans la pulsation point la pulsion, entendue au sens psychanalytique d'une poussée qui trouve son origine dans un état de tension somatique et qui, en s'attachant à un objet, tend vers son but : la suppression de cette tension ${ }^{7}$. L'exploration de cette dynamique où pulsation et pulsion s'intriquent permet d'inscrire les sculptures cinétiques de Horn dans la continuité de ses performances. Car le corps, chez Horn, n'est pas simplement une machine - l'analogie serait banale -, il est, pour reprendre l'expression de Gilles Deleuze et Félix Guattari ${ }^{8}$, une " machine désirante " : la matrice des pulsions.

Le travail sur la pulsion et son inhibition est au cœur de l'œuvre de Horn. Dans nombre d'entretiens, l'artiste situe à l'origine de son travail une expérience traumatisante : alors qu'elle était étudiante en école d'art, elle fit une intoxication respiratoire à la suite d'un travail avec des matériaux toxiques. Cette intoxication grave lui valut de passer un an dans une clinique, puis un sanatorium - une année de maladie et de réclusion à laquelle elle se confronte dans ses performances. Horn explique :

Wenn man isoliert oder alleine ist, hat man eine starke Sehnsucht nach Kontakten und den Wunsch, sich über seinen Körper mitzuteilen. Wenn man sich meine frühen Arbeiten ansieht, entdeckt man immer eine Art Kokon, durch den ich mich selbst zu schützen versuchte, wie zum Beispiel die Fächer, in die ich mich einschließen und isolieren konnte, die ich aber auch wieder öffnen und so eine andere Person in ein intimes Ritual einbeziehen konnte ${ }^{9}$.

7. « Das Ziel eines Triebes ist allemal die Befriedigung, die nur durch Aufhebung des Reizzustandes an der Triebquelle erreicht werden kann ", Sigmund Freud, Triebe und Triebschicksale (1915), in Gesammelte Werke, t. X, Frankfurt a. M., Fischer, 1991, p. 215.

8. Cf. Gilles Deleuze, Félix Guattari, L’Anti-CEdipe. Capitalisme et schizophrénie, Paris, Éditions de Minuit, 1972.

9. Rebecca Horn, entretien avec Germano Celant, in Rebecca Horn, Ostfildern, Hatje Cantz, 1993, p. 24. 
Loin de rompre l'isolement, les performances de Rebecca Horn s'appuient sur l'empêchement de la rencontre amoureuse : c'est dans la tension entre l'élan et son inhibition que le désir devient visible. Les " extensions corporelles " sont des parures qui, par leur beauté ou leur bizarrerie, attirent le regard du spectateur, mais ce sont aussi des paravents, des cocons, des armures, derrière lesquels le corps se retire. Elles sont érotiques dans le sens où l'entend Barthes : "L'endroit le plus érotique d'un corps n'est-il pas là où le vêtement bâille? [...] c'est l'intermittence [...] qui est érotique : celle de la peau qui scintille entre deux pièces [...] ; c'est ce scintillement même qui séduit, ou encore : la mise en scène d'une apparition-disparition ${ }^{10}$."

La parade nuptiale du paon est l'un des leitmotivs du travail de Horn, qui fait, justement, du mouvement de déploiement et de repli des plumes " la mise en scène d'une apparition-disparition ". L'une des premières élaborations sur ce motif est la performance Paradieswitwe (Veuve de paradis, 1975), où le corps nu de la performeuse, d'abord dissimulé dans un long cocon de plumes noires, est partiellement dévoilé par l'écartement des bandeaux de plumes qu'elle manipule. Le motif est repris dans le film Der Eintänzer (Le taxi-danseur, 1978), mais dans une dynamique inverse, cette fois : la jeune femme, une ballerine, disparaît lentement dans un double éventail de plumes blanches. Le moment le plus beau - celui où les plumes forment une roue complète - est aussi celui où le corps érotisé devient inaccessible. Horn le répète : " Il s’agit du fait d'être séparé - d'une façon qui n'est même pas si désagréable que ça - de l'objet du désir ${ }^{11}$. " Voir ou toucher, admirer ou posséder : en rendant sensible cette alternative, les performances de Horn mettent à l'épreuve du désir la thèse kantienne de la "satisfaction désintéressée ${ }^{12}$ " sur laquelle doit se fonder l'expérience esthétique. L'art, semble demander Horn, ne tiendrait-il pas justement son pouvoir de fascination de sa capacité à mobiliser le désir sans le satisfaire, à susciter une frustration voluptueuse? Ces performances où les " extensions corporelles " permettent au corps humain de séduire, d'attirer le regard à soi, sont toujours habitées par l'angoisse que suscitent le passage à l'acte, la possibilité de l'assouvissement du désir et donc de l'épuisement de la tension. Le corps humain y est à la fois éminemment visible - souvent, d'ailleurs, nu - et inaccessible, maintenu à distance par les harnais, les masques et les éventails. Avec la levée de la frontière entre les corps, c'est la " petite mort " qui menace, et c'est l'angoisse de la perte dans l'autre que les " extensions corporelles " sont tenues de conjurer.

Le mouvement de va-et-vient qui structure l'essentiel des performances de Horn, s'il donne d'abord l'image des processus organiques, devient bientôt la mise en scène de la pulsion sexuelle et de la charge d'angoisse qu'elle

10. Roland Barthes, Le Plaisir du texte, Paris, Le Seuil, 1973, p. 17-18.

11. "Es dreht sich um das Getrennt-Sein - in einer gar nicht einmal so unangenehmen Weise - vom Objekt der Begierde ", Rebecca Horn, entretien avec Germano Celant, Rebecca Horn, p. 25 (c'est nous qui traduisons).

12. "Das Wohlgefallen, welches das Geschmacksurtheil bestimmt, ist ohne alles Interesse ", Immanuel Kant, Kritik der Urtheilskraft, \$2 (1790), Frankfurt a. M., Suhrkamp, 1974, p. 116 (c'est nous qui traduisons). 
véhicule. L'alternance entre l'exhibition et la dissimulation, le rapprochement et l'écartement sont les mouvements du désir lui-même, mouvements que les sculptures cinétiques répètent indéfiniment, avec une lenteur fascinante. Les moments de suspension, les petites résistances qui viennent de l'imperfection du mécanisme donnent véritablement vie à ces mouvements : les machines sont comme troublées par leur propre désir, semblent manifester des hésitations, de la fatigue ou de la crainte. Horn insiste souvent sur la vie de ses " machines désirantes " : "Le spectateur est perturbé par l'autonomie manifestée par les machines avec leurs mouvements irréguliers, parce qu'il a pénétré dans un monde vivant où la communication bat déjà son plein et où il a d'abord le sentiment d'être un intrus ${ }^{13}$. "Claudia Bahmer remarque à juste titre que " les objets anthropomorphiques de Horn, qui jouent un rôle de médiateurs et, par moment, d'adversaires des protagonistes humains apparemment "réifiés", se présentent comme des métaphores de la vulnérabilité de l'existence humaine [...] ${ }^{14}$ ". Les machines de Horn nous semblent d'autant plus vivantes que nous les surprenons dans des moments d'intimité : tentatives d'approche, caresses, pénétrations et, rarement, orgasmes. Le spectateur transformé en voyeur, hypnotisé par les rituels de séduction des machines, est confronté aux ressorts de son propre désir. Si ces machines sont si fascinantes, c'est qu'elles en disent long, dans la forme et le rythme intermittent de leurs mouvements, sur ce mélange d'élan et d'appréhension, d'impulsion et d'inhibition, de douceur et d'agressivité qui constitue le désir. En donnant à voir le désir à partir du mouvement et du rythme, Horn le dépouille de la dimension sentimentale qui lui est souvent associée dès qu'il s'agit du désir proprement humain. À cet égard aussi, elle met au jour une dynamique - et plus précisément, une intentionnalité - qui n'est pas de l'ordre du sentiment ou du projet, mais de celui de la pulsion - une intentionnalité qui, à ce titre, ne trouve pas son origine dans la subjectivité, mais dans le corps.

\section{Art sans sujet}

Horn privilégie l'automatisme face au mouvement expressif, c'est-à-dire au mouvement motivé par une intériorité, que cette intériorité soit identifiée à la conscience et à ses projets ou au siège des sentiments. Dans le va-et-vient, elle découvre une dynamique fondamentale, commune à tout ce qui se meut,

13. "The viewer is disturbed by the autonomy manifested by the machines with their irregular movements, because he has entered a living world where communications is already in full swing and where he initially feels like an intruder ", Rebecca Horn (propos traduits), entretien avec Carl Haenlein, in Rebecca Horn. The Glance of Infinity, p. 16.

14. "Vielmehr können Horns anthropomorphe Objekte, die als Mittler und zuweilen auch als Gegenspieler der äußerlich "verdinglichten" menschlichen Protagonisten erscheinen, als Metapher für die Verletzlichkeit jeglichen menschlichen Daseins gelten [...] ", Claudia Bahmer " "Melancholische Darsteller in völliger Einsamkeit". Rebecca Horns anthropomorphe Objekte als Kehrseite des künstlichen Menschen ", in Gisela Febel, Cerstin Bauer-Funke (dir.), Menschenkonstruktionen: künstliche Menschen in Literatur, Film, Theater und Kunst des 19. und 20. Jahrhunderts, Göttingen, Wallstein, 2004, p. 148-168, ici p. 168. 
qu'elle charge d'une dimension pulsionnelle, libidinale, mais non sentimentale. L'intérêt qu'elle porte à la spontanéité dans ce qu'elle a de proprement somatique permet à Horn de trouver sa place dans une tradition "dissidente" de l'esthétique allemande, qui accorde un rôle central à l'involontaire, sans pour autant recourir au topos de l'inspiration ou du sentiment. Cette tradition n'est en ce sens ni celle du classicisme, ni celle du romantisme : elle s'inscrit bien plutôt dans la lignée de l'essai de Kleist Über das Marionettentheater $(1810)^{15}$. Si cet essai est surprenant, c'est qu'il prend le contre-pied tant de la tradition esthétique fondée sur la maîtrise formelle que de celle qui fait valoir la spontanéité du sentiment. Schiller, dans son traité Über Anmut und Würde (1793), livre le modèle de cette esthétique de l'état d'âme contre laquelle Kleist, implicitement, se positionne. Schiller y fait de la grâce un mouvement involontaire, mais où s'exprime la disposition morale d'une personne :

Eine willkürliche Bewegung, wenn sie sich nicht zugleich mit einer sympathetischen verbindet oder, was ebensoviel sagt, nicht mit etwas Unwillkürlichem, das in dem moralischen Empfindungszustand der Person seinen Grund hat, vermischt, kann niemals Grazie zeigen, wozu immer ein Zustand im Gemüt als Ursache erfordert wird ${ }^{16}$.

La grâce ne peut émaner que d'un mouvement où s'harmonisent la nature et l'âme, le corps et l'" état affectif moral ", et, en ce sens, seul le mouvement expressif - celui où c'est toute la personne morale qui se révèle dans le geste peut être esthétique. Kleist, dans l'essai qu'il consacre au théâtre de marionnettes, soutient la thèse provocante que le mouvement le plus gracieux est celui des pantins, parce que ceux-ci sont totalement dépourvus de conscience comme d'âme et n'obéissent qu'aux règles physiques régissant la matière :

Und der Vorteil, den diese Puppe vor lebendigen Tänzern voraus haben würde?

Der Vorteil? Zuvörderst ein negativer, mein vortrefflicher Freund, nämlich dieser, daß sie sich niemals zierte. - Denn Ziererei erscheint, wie Sie wissen, wenn sich die Seele (vis motrix) in irgend einem andern Punkte befindet, als in dem Schwerpunkt der Bewegung. Da der Maschinist nun schlechthin, vermittelst des Drahtes oder Fadens, keinen andern Punkt in seiner Gewalt hat, als diesen: so sind alle übrigen Glieder, was sie sein sollen, tot, reine Pendel, und folgen dem bloßen Gesetz der Schwere; eine vortreffliche Eigenschaft, die man vergebens bei dem größesten Teil unsrer Tänzer sucht ${ }^{17}$.

La marionnette devra toujours surpasser l'être humain, parce qu'en elle le mouvement n'est pas perturbé par la volonté, qui cherche à se soumettre la spontanéité corporelle, ni par le sentiment ou la disposition morale, qui détournent l'attention du corps pour l'orienter vers une intériorité que le geste est censé exprimer. La marionnette de Kleist donne l'image d'un corps affranchi de la subjectivité, qui n'est donc plus tenu de se faire le véhicule d'une intention ou d'un sentiment, mais vaut pour lui-même.

15. Lynne Cooke évoque le rapprochement avec l'esthétique kleistienne dans " Horn's Sensorium: Site and Source of Desire ", in Rebecca Horn. The Glance of Infinity, p. 21-29, ici p. 28, note 10.

16. Friedrich Schiller, Über Anmut und Würde (1793), Freiburg im Breisgau, Die Ordnung, 1948, p. 24. C'est Schiller qui souligne.

17. Friedrich von Kleist, Über das Marionettentheater (1810), in Der Zweikampf... und andere Prosa, Stuttgart, Reclam, 2002, p. 82. 
Le projet de donner au mouvement non-expressif une valeur esthétique, et une place sur la scène artistique, a mobilisé les avant-gardes dès le début du $\mathrm{XX}^{\mathrm{e}}$ siècle. Cette contestation de la préséance de l'âme, du sentiment, sur l'énergie somatique s'est d'abord articulée dans la fronde des artistes du mouvement Dada contre l'expressionnisme. Que l'on songe à la diatribe de Richard Huelsenbeck dans le " Dada Manifesto " (1918) : " Sous prétexte de propager l'âme, [les expressionnistes], dans leur combat contre le naturalisme, ont rouvert la voie à une gestuelle pathétique et abstraite, qui présuppose une vie dépourvue de contenu, confortable et immobile ${ }^{18}$. "Des lectures-performances d'Hugo Ball au " théâtre épique " de Bertolt Brecht, la rupture avec la gestuelle pathétique de l'expressionnisme prend différentes formes. Mais s'il est possible de trouver, parmi ces mouvements de renouvellement scénique, un précurseur de Horn, il s'agit certainement du décorateur de théâtre et scénographe Oskar Schlemmer, rattaché au Bauhaus, qui lui aussi voulut renouer le lien entre mouvement vital et mouvement mécanique. Son Triadisches Ballett (1922) met en scène des poupées mécaniques, marionnettes et robots étrangement vivants et touchants : l'usage des encombrants costumes imaginés par Schlemmer - costumes qui annoncent à certains égards les " extensions corporelles " de Horn - limite la liberté de mouvement des danseurs et danseuses, pour mieux leur permettre d'adopter des gestes rudimentaires, qui, par leur simplicité, leur répétitivité mêmes, fascinent et troublent ${ }^{19}$. Un demi-siècle après Schlemmer, Horn réaffirme à son tour la dignité esthétique du mouvement machinal, qui, dans sa forme et son rythme, rappelle que le corps "propre ", le corps que le sujet reconnait comme sien, ne peut être éprouvé qu'au prix de l'oubli de cette vie somatique et pulsionnelle qui constitue l'» impropre " de l'homme.

Mais Horn fait plus que de faire accéder à la représentation cette vie trop souvent ignorée, méprisée, elle lui prête également un potentiel créateur. Dans la performance Bleistiftmaske (Masque-crayons, 1972) mobilisant l'» extension corporelle " du même nom, l'artiste explore le lien entre contrainte, compulsion et création. L'artiste y porte un masque de lanières noires sur lesquelles sont fichés des crayons à papier : approchant son visage d'un mur blanc, elle y trace des lignes noires dans un mouvement répété de va-et-vient. Les lanières hérissées de crayons qui couvrent le visage suggèrent d'abord le lien entre la sublimation et la coercition, comme le faisait déjà, plus discrètement, Einhorn. Le " masque-crayons ", comme les « doigts-gants " de la performance Mit beiden

18. «Unter dem Vorwand, die Seele zu propagieren, haben [die Expressionisten] im Kampfe gegen den Naturalismus zu den abstraktpathetischen Gesten zurückgefunden, die ein inhaltloses, bequemes und unbewegtes Leben zur Voraussetzung haben ", Richard Huelsenbeck, "Dada Manifesto " (1918), http://germanhistorydocs.ghi-dc.org/pdf/deu/dada_ger.pdf [dernière consultation le 7 juillet 2018] (c'est nous qui traduisons).

19. Alexandra Tacke (Rebecca Horn. Künstlerische Selbstpositionierungen im kulturellen Raum, p. 21-28) et Nancy Spector ("Weder Junggesellen noch Bräute. Die hybriden Maschinen der Rebecca Horn ", in Rebecca Horn, p. 65-79) reviennent sur la place de Horn dans cette tradition esthétique qui s'intéresse à la machine, notamment sur l'influence qu'exerce sur elle le travail des surréalistes (Meret Oppenheim, Marcel Duchamp, Man Ray...). 
Händen gleichzeitig die Wände berühren, joue de la tension entre l'extension spatiale du corps et son isolement. Par ailleurs, la performance Bleistiftmaske met en scène le rôle des pulsions dans le processus créateur : dans le "masque-crayons " - inquiétant instrument de torture et de mortification -, la pulsion de mort, qui s'expose dans sa double détermination masochiste et sadique, donne son élan à la sublimation. Mais, ironiquement, Horn bloque cet élan, empêche que le détournement de la pulsion vers l'art aboutisse pleinement : le geste créateur, réduit au va-et-vient, révèle sa dimension compulsionnelle. En faisant basculer la pulsion dans la compulsion, dans la " contrainte de répétition " (Freud), Horn tient à distance la référence nietzschéenne au " dionysiaque " : le déraillement du pulsionnel dans le pathologique menace constamment et empêche de livrer une interprétation vitaliste de ses œuvres.

Dès lors qu'il s'agit de mettre en lumière l'origine pulsionnelle, voire compulsionnelle du geste créateur, il n'est pas surprenant qu'ici aussi la machine puisse prendre la place de la performeuse. Horn poursuit ce travail de mise à nu avec les Malmaschinen (Machines à peindre) créées au début des années 1990 : ces machines tantôt actionnées par des mouvements brusques de la main, tantôt automatiques, rappellent la série de sculptures cinétiques Méta-Matics que Jean Tinguely construit dans les années 1950. Comme leurs aînées, les Malmaschinen mobilisent leurs mécanismes pour créer des œuvres non pas expressives, mais impulsives. Elles donnent à Horn l'occasion de poursuivre son exploration du mouvement pulsionnel, pour y chercher le point d'articulation de la destruction et de la création. Dans l'installation High Moon (1991), comme dans Bleistiftmaske, le désir fait la part belle à la pulsion de mort : deux fusils braqués l'un vers l'autre, et alimentés d'un liquide rouge contenu dans deux entonnoirs dont les courbes douces évoquent des seins, tirent leur munition de liquide et éclaboussent les murs opposés de jaillissements pourpres. Les éclaboussures des machines-artistes, qui suggèrent tout à la fois le sang, le sperme et le lait maternel, rappellent que la création - et le plaisir troublant qu'elle procure à ses spectateurs - puise dans un fond pulsionnel, qui lui-même trouve son origine dans une vie somatique que le sujet ne peut s'assujettir.

En réévaluant le mouvement pulsionnel, Rebecca Horn détourne le regard de l'âme, de la conscience, de l'intériorité, pour l'attacher à la spontanéité corporelle : dans ses performances, le corps " propre " (Leib) devient un corps étranger (Körper) - un corps dont la dynamique est irréductible aux projets du sujet, comme à l'expression de ses sentiments. Loin de chercher à dissiper l'opacité de cette vie somatique, elle donne à voir les mouvements et les rythmes les plus élémentaires de ces processus vitaux trop vite oubliés, refoulés par un sujet pressé de se croire "maître dans sa propre maison ${ }^{20}$ ". Ces performances, exécutées par l'être humain ou la machine, rappellent ainsi que le corps a longtemps été rejeté hors du champ esthétique en raison de la "blessure narcissique" qu'il inflige à

20. “ Herr im eigenen Haus ", Freud, Une difficulté de la psychanalyse, p. 50. 
l'homme : en raison des liens transgressifs qui s'y révèlent entre l'humain et l'animal, et, si l'on pousse plus loin, entre le mouvement vital et le mouvement machinal. À travers la mise au jour du lien génétique entre pulsation et pulsion, pulsion et compulsion, les performances de Rebecca Horn donnent un droit de cité artistique au corps, à ses mécanismes et à ses machinations. 